\title{
Pressure-induced quantum phase transition in the quantum antiferromagnet $\mathrm{CsFeCl}_{3}$
}

\author{
Shohei Hayashida, ${ }^{1}$ Oksana Zaharko, ${ }^{2}$ Nobuyuki Kurita, ${ }^{3}$ Hidekazu Tanaka, ${ }^{3}$ Masato Hagihala, ${ }^{1}$ Minoru Soda,,${ }^{1}$ Shinichi Itoh, ${ }^{4}$ \\ Yoshiya Uwatoko, ${ }^{1}$ and Takatsugu Masuda ${ }^{1}$ \\ ${ }^{1}$ Institute for Solid State Physics, The University of Tokyo, Chiba 277-8581, Japan \\ ${ }^{2}$ Laboratory for Neutron Scattering and Imaging, Paul Scherrer Institut, CH-5232 Villigen, Switzerland \\ ${ }^{3}$ Department of Physics, Tokyo Institute of Technology, Meguro-ku, Tokyo 152-8551, Japan \\ ${ }^{4}$ Neutron Science Division, Institute of Materials Structure Science, High Energy Accelerator Research Organization, \\ Tsukuba, Ibraki 305-0801, Japan
}

(Received 28 February 2018; revised manuscript received 18 April 2018; published 30 April 2018)

\begin{abstract}
We have studied the pressure-induced quantum phase transition in the singlet-ground-state antiferromagnet $\mathrm{CsFeCl}_{3}$. Neutron diffraction experiments under pressure evidence the magnetic long-range order at low temperatures. Magnetic structure analysis reveals a $120^{\circ}$ structure with a propagation vector of $\boldsymbol{k}_{\mathrm{mag}}=(1 / 3,1 / 3,0)$. The estimated critical exponent of the order parameter suggests that $\mathrm{CsFeCl}_{3}$ belongs to the universality class of $U(1) \times Z_{2}$ symmetry which is expected to realize the chiral liquid state.
\end{abstract}

DOI: 10.1103/PhysRevB.97.140405

Quantum phase transition (QPT) rooted in the quantum uncertainty between kinetic and potential energies has been a central topic of condensed-matter physics. The extent of the quantum fluctuation determines the ground state to be or not to be ordered, and it is controlled by external parameters such as pressure, magnetic field, and chemical doping [1,2]. One has a chance to observe an exotic phenomenon in the vicinity of the quantum critical point (QCP) in which the effect of the quantum fluctuation is enhanced. To date the QPT has provided unsolved puzzles in many condensed-matter systems including quantum spin systems [3,4], high-temperature superconductors [5-8], heavy fermion metals $[9,10]$, two-dimensional electron systems [11], and ultracold atom systems [12,13].

The importance of the quantum spin system is an easy access to the verification of experiments and theories. For instance, in the spin-dimer compound $\mathrm{TlCuCl}_{3}$, the QPTs induced by the magnetic field [14,15] and the pressure [16-19] have been observed. These experimental works have triggered many theoretical studies: the interpretation of the field-induced order as the Bose-Einstein condensation of magnons [20-22], the Higgs mode near the QCP $[23,24]$, and universality in threedimensional (3D) quantum antiferromagnets (AFs) [25-29]. The spin system is thus a good playground for the study of the QPT.

The spin $S=1$ AF of easy-plane type is one of the simplest systems exhibiting the QPT. The Hamiltonian is expressed by

$$
\mathcal{H}=\sum_{i} D\left(S_{i}^{z}\right)^{2}+\sum_{i j} J_{i j} S_{i} \cdot S_{j},
$$

where $S_{i}$ is a spin operator at the $i$ th site. The easy-plane anisotropy $D$ splits the triplet spin $S=1$ into the singlet $S^{z}=0$ and the doublet $S^{z}= \pm 1$ as shown in Fig. 1(a), favoring the disordered singlet ground state. In contrast, the spin interaction $J_{i j}$ favors a magnetic order. Control of the competition between the anisotropy and the spin interaction by an external parameter brings about the QPT. Recent theories predict nontrivial phenomena near the QCP: an enhanced longitudinal magnon in the tetragonal lattice [30] and the appearance of the chiral liquid state in the triangular lattice [31]. From the viewpoint of experiment, hydrostatic pressure is a good external parameter since it tunes the spin interaction and anisotropy with the system symmetry unchanged. It is noted that applying magnetic field lowers the symmetry and chemical doping induces randomness. Nevertheless, pressure-induced QPT has not been experimentally studied in the easy-plane type $\mathrm{AF}$ having the singlet ground state.

$\mathrm{CsFeCl}_{3}$ is a singlet-ground-state $\mathrm{AF}$ where $\mathrm{Fe}^{2+}$ ions $\left(3 d^{6}\right.$, $S=2, L=2$ ) are responsible for the magnetism. The $\mathrm{FeCl}_{6}$ octahedra form a one-dimensional chain along the crystallographic $c$ axis as shown in Fig. 1(b), and the chains form the triangular lattice in the $a b$ plane in Fig. 1(c) [32]. The lowenergy excitation of the $\mathrm{Fe}^{2+}$ ion is described by a pseudospin $s=1$ due to the cubic crystal field and spin-orbit coupling. Moreover, the pseudospin is split into $s^{z}=0$ singlet ground state and $s^{z}= \pm 1$ doublet state caused by the easy-plane type single-ion anisotropy $D\left(s^{z}\right)^{2}$. Inelastic neutron scattering experiments identified the spin system as the ferromagnetic chain which is weakly coupled by the antiferromagnetic interaction in the triangular plane [33]. Combination of the low dimensionality and the strong easy-plane-type anisotropy favors the quantum singlet ground state. Indeed, the magnetic order was not observed at low temperatures [34].

Very recently, the magnetic susceptibility measurement exhibited a pressure-induced magnetic transition at a low temperature [35]. The result indicated that applying pressure effectively enhanced the spin interaction, suppressed the anisotropy gap, and induced a QPT. $\mathrm{CsFeCl}_{3}$ is thus a good model compound for the pressure-induced QPT in the spin $S=1$ singlet-ground-state AF. It is of primary importance to identify the magnetic structure and to discuss the universality class of the pressure-induced ordered phase in $\mathrm{CsFeCl}_{3}$.

A single-crystal sample was grown by the vertical Bridgman method [35]. The crystal with the mass of $0.20 \mathrm{~g}$ was put in a 

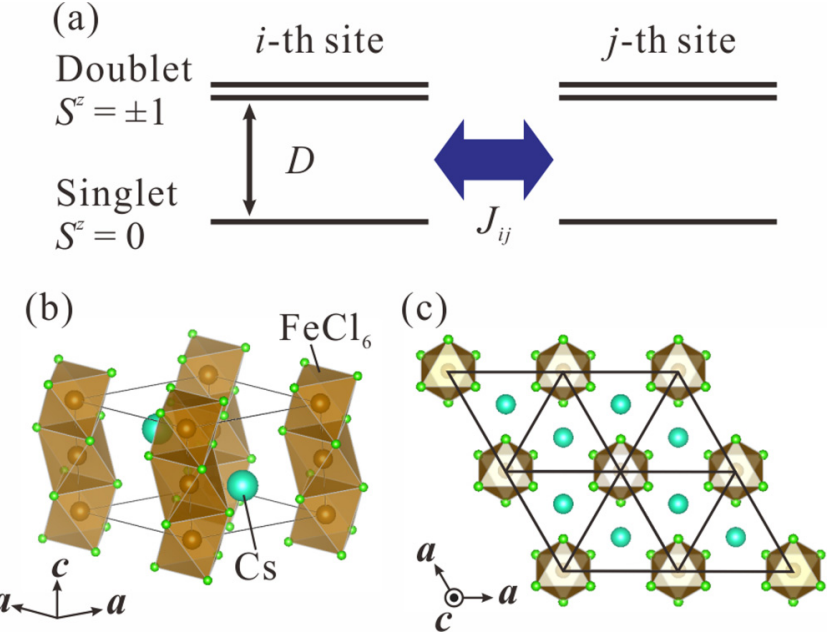

FIG. 1. (a) Schematic diagram of local energy scheme and intersite interaction. (b) Perspective view of the crystal structures of $\mathrm{CsFeCl}_{3}$ with the space group $P 6_{3} / m m c$. (c) Triangular lattice in $\mathrm{CsFeCl}_{3}$.

Teflon cell with a deuterated glycerin as a pressure medium. The crystal was mounted so that the horizontal plane was the $a b$ plane. The Teflon cell was installed in a piston-cylinder clamped cell made of $\mathrm{Al}$ alloy. The pressure-induced magnetic order was observed by a preliminary neutron Laue diffraction measured at the high-resolution chopper spectrometer (HRC) in J-PARC/MLF, Japan. Then neutron diffraction experiments under pressures were performed at the single-crystal neutron diffractometer ZEBRA at the Swiss Neutron Spallation Source, Paul Scherrer Institut (PSI) in Switzerland. A ${ }^{4} \mathrm{He}$ cryostat was used to achieve the base temperature of $1.6 \mathrm{~K}$. A Ge(311) monochromator was chosen to obtain the neutrons with the wavelength of $1.178 \AA$. In addition to the in-plane reflections of $(h, k, 0)$, the out-of-plane reflections of $(h, k, \pm 1)$ were collected by using the lifting counter of ZEBRA. The data were analyzed by the Rietveld method using FULLPROF software [36]. Candidates for the magnetic structure compatible with the lattice symmetry were obtained by the SARA $h$ software [37].

The $\omega$ scans at $\boldsymbol{Q}=(1 / 3,1 / 3,0)$ reflection measured at $1.6 \mathrm{~K}$ for $P=0.0$ and $2.0 \mathrm{GPa}$ are shown in Fig. 2. A new peak is induced by applying pressure. Its intensity increases with the decrease of the temperature below $5 \mathrm{~K}$ as shown in the inset of Fig. 2, which means that the peak is magnetic. The magnetic Bragg peaks are observed in $(h \pm 1 / 3, k \pm 1 / 3,0)$, leading to the magnetic propagation vector of $\boldsymbol{k}_{\mathrm{mag}}=(1 / 3,1 / 3,0)$.

The nuclear Bragg peaks at each pressure and at the base temperature are reasonably fitted by the hexagonal structure with the space group $P 6_{3} / m m c$ which was previously reported for the room temperature [32]. The $R_{\mathrm{F}}$ factors for 1.4, 1.6, 1.8, 2.0, and $2.2 \mathrm{GPa}$ are 8.2, 4.5, 3.8, 13.2, and 5.2\%. We can thus safely assume that the crystal structure with the space group $P 6_{3} / m m c$ is retained at each pressure in the magnetic structure analysis. In the representation analysis, the space group $P 6_{3} / \mathrm{mmc}$ and the magnetic propagation vector $\boldsymbol{k}_{\mathrm{mag}}=(1 / 3,1 / 3,0)$ lead to four irreducible representations $\Gamma_{3}+\Gamma_{4}+\Gamma_{5}+\Gamma_{6}$. The basis vectors for $\Gamma_{3}$ and $\Gamma_{4}$ exhibit the easy-axis type structures with the magnetic moments along the

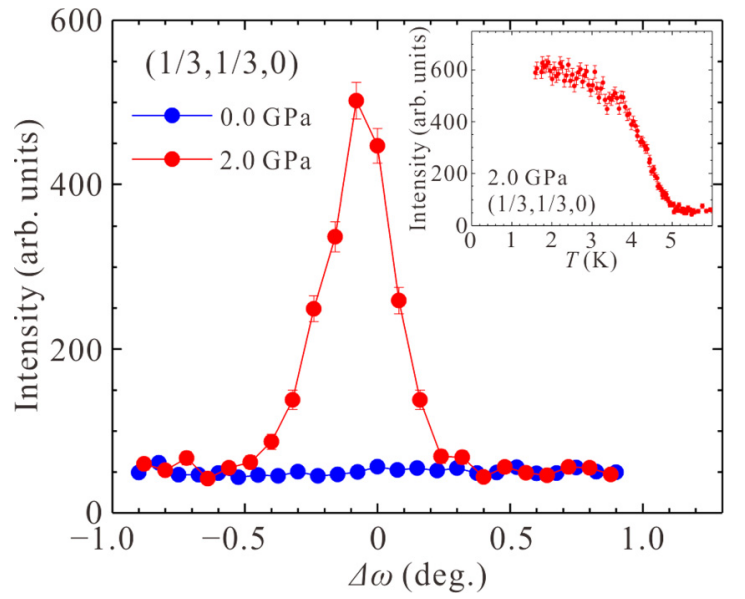

FIG. 2. The $\omega$ scans for $(1 / 3,1 / 3,0)$ reflection measured at $T=1.6 \mathrm{~K}$. The blue and red circles are data at 0.0 and $2.0 \mathrm{GPa}$, respectively. The inset is the temperature dependence of the magnetic Bragg peak intensity for $(1 / 3,1 / 3,0)$ reflection measured at $2.0 \mathrm{GPa}$.

$c$ axis in which the moments are modulated by the propagation vector. The basis vectors for $\Gamma_{5}$ and $\Gamma_{6}$ exhibit $120^{\circ}$ structures in the $a b$ plane. The relations of the neighboring moments along the $c$ axis are ferromagnetic for $\Gamma_{3}$ and $\Gamma_{6}$, and those are antiferromagnetic for $\Gamma_{4}$ and $\Gamma_{5}$.

The magnetic Bragg peaks are observed in the case of $l=0$ and not in the case of $l=1$ as listed in Table I, which means that the alignment of the moments along the $c$ axis is ferromagnetic. Then the models of $\Gamma_{4}$ and $\Gamma_{5}$ are excluded. Calculated neutron intensities at the $l=0$ reflections for $\Gamma_{3}$ and $\Gamma_{6}$ are the same. Consider that $\mathrm{CsFeCl}_{3}$ has the easy-plane type anisotropy, the model of the $\Gamma_{3}$ exhibiting easy-axis type structure is not reasonable, and the model of the $\Gamma_{6}$ is selected. As the result of the Rietveld refinement in Fig. 3, the structure for $\Gamma_{6}$ gives a good agreement with the experimental data. The magnetic moments therefore form the $120^{\circ}$ structure in the $a b$ plane, and ferromagnetically propagate along the $c$ axis as shown in the inset of Fig. 3. The refined magnitude of the moment and refinement factor for each pressure are listed in Table II.

To discuss the universality class of the pressure-induced ordered phase, we estimate the critical exponent of the order parameter. Unlike a typical second-order transition, the order parameter exhibits a broad transition as shown in the inset of Fig. 2. We presume that the inhomogeneity of the pressure leads to the distribution of the transition temperature $T_{\mathrm{N}}$ [38-41]. Then the temperature dependence of the magnetic peak is

TABLE I. Integrated intensities of the magnetic Bragg peaks at $1.6 \mathrm{~K}$ and at $2.0 \mathrm{GPa}$.

\begin{tabular}{lc}
\hline \hline$(h, k, l)$ & $|F|^{2}$ (arb. units) \\
\hline$(1 / 3,1 / 3,0)$ & 87.06 \\
$(2 / 3,2 / 3,0)$ & 43.39 \\
$(4 / 3,-1 / 3,0)$ & 41.88 \\
$(1 / 3,1 / 3,1)$ & 0 \\
$(2 / 3,2 / 3,1)$ & 0 \\
$(4 / 3,-1 / 3,1)$ & 0 \\
\hline \hline
\end{tabular}




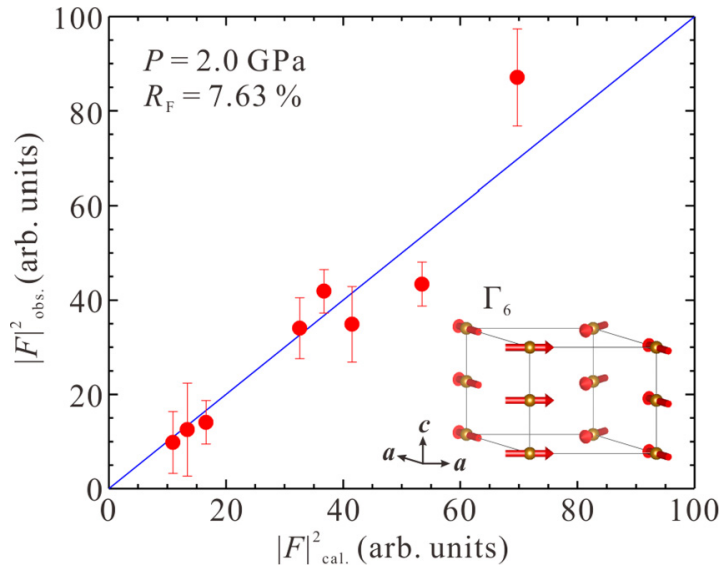

FIG. 3. Magnetic structure factors measured at $1.6 \mathrm{~K}$ and at 2.0 GPa plotted against those obtained by the model calculation for $\Gamma_{6}$. The inset is an example of the magnetic structure for $\Gamma_{6}$.

expressed by

$$
\begin{aligned}
I(T)= & I_{0}+A \sqrt{\frac{4 \ln (2)}{\pi \Delta T_{\mathrm{N}}^{2}}} \int \exp \left(-4 \ln (2) \frac{\left(\tau-T_{\mathrm{N}}\right)^{2}}{\Delta T_{\mathrm{N}}^{2}}\right) \\
& \times(\tau-T)^{2 \beta} d \tau,
\end{aligned}
$$

where $I_{0}$ is offset, $A$ is the scaling factor of the intensity, $\Delta T_{\mathrm{N}}$ is the fluctuation of $T_{\mathrm{N}}$, and $\beta$ is the critical exponent. $\Delta T_{\mathrm{N}}$ is fixed to be $0.4 \mathrm{~K}$, since the accuracy of applying pressure is $\pm 0.1 \mathrm{GPa}$ and the $T_{\mathrm{N}}$ shifts by $0.4 \mathrm{~K}$ with the change of $0.1 \mathrm{GPa}$ in the pressure-temperature phase diagram [35]. We perform the fitting to the data at $2.2 \mathrm{GPa}$ because the $T_{\mathrm{N}}$ is the highest and the number of data points is the most among the measured pressures. The data in the range of $4.5 \mathrm{~K}<T<$ $7.0 \mathrm{~K}$ are used for the fit, where the $I_{0}$ is evaluated from the high-temperature data. Fitting parameters are $T_{\mathrm{N}}, A$, and $\beta$. As a result of the fitting, the transition temperature $T_{\mathrm{N}}=5.6$ $\mathrm{K}$ and the critical exponent $\beta=0.24(1)$ are obtained. Using the value of the critical exponent, the $T_{\mathrm{N}}$ of other pressures are refined. The refined $T_{\mathrm{N}}$ 's are listed in Table II.

The temperature evolution of the order parameters is shown in Fig. 4. The scaled temperatures $t=T / T_{\mathrm{N}}$ are used. The magnitude of the moment at each pressure is scaled by that at $t=0.56$ which corresponds to the base temperature of the data at $1.4 \mathrm{GPa}$. The order parameters for all the pressures yield to the same curve, indicating that the universality class of the ordered phase is independent on pressure.

The obtained exponent $\beta=0.24(1)$ is close to $\beta=0.253$ which is the theoretical prediction for an $X Y$ stacked triangular

TABLE II. The estimated $T_{\mathrm{N}}$ and magnitude of the magnetic moment $|M|$ at each pressure.

\begin{tabular}{lccc}
\hline \hline$P(\mathrm{GPa})$ & $T_{\mathrm{N}}(\mathrm{K})$ & $|\boldsymbol{M}|\left(\mu_{\mathrm{B}}\right)$ & $R_{\mathrm{F}}(\%)$ \\
\hline 1.4 & 2.8 & $2.63(4)$ & 7.23 \\
1.6 & 3.8 & $2.87(5)$ & 7.62 \\
1.8 & 4.5 & $3.10(6)$ & 10.1 \\
2.0 & 4.7 & $3.16(5)$ & 7.63 \\
2.2 & 5.6 & $3.15(2)$ & 3.47 \\
\hline \hline
\end{tabular}

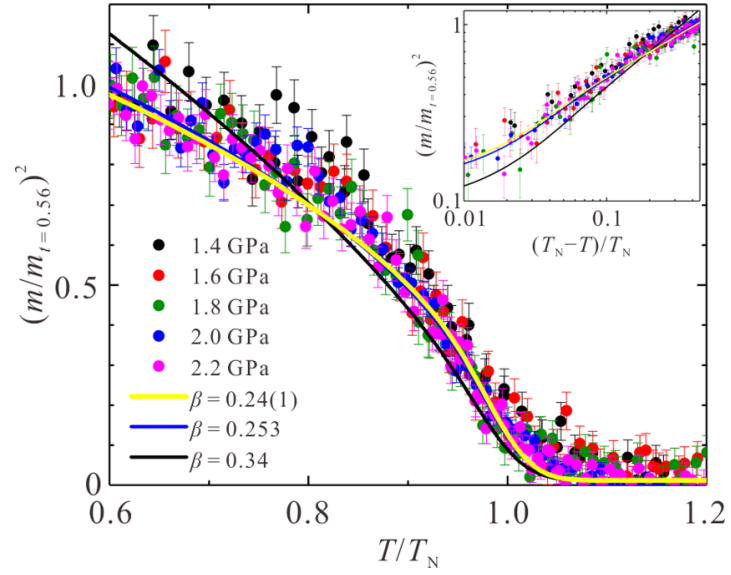

FIG. 4. Temperature evolution of the order parameter for each pressure. The temperatures are scaled by $t=T / T_{\mathrm{N}}$. The order parameters are scaled by the magnitudes of the moments at $t=0.56$. Yellow, blue, and black solid curves indicate calculation curves having the critical exponents $\beta$ of $0.24(1), 0.253$, and 0.34 , respectively. The inset is the log-log of $\left(m / m_{t=0.56}\right)^{2}$ vs $\left(T_{\mathrm{N}}-T\right) / T_{\mathrm{N}}$.

AF (STA) $[42,43]$. The temperature dependences of the order parameter using critical exponents $\beta=0.253$ for the $X Y$ STA model and $\beta=0.34$ for the 3D $X Y$ model [44] are indicated by the blue and black solid curves in Fig. 4. The parameters other than $\beta$ are the same as those at $2.2 \mathrm{GPa}$. Compared with the theoretical curves, the $X Y$ STA model is more reasonable than the 3D $X Y$ model. Considering the lattice symmetry and the strong easy-plane anisotropy [33], we can safely conclude that the universality class in $\mathrm{CsFeCl}_{3}$ belongs to the $X Y$ STA model. So far, the universality class of the Heisenberg or $X Y$ STA model has been reported in $\mathrm{CsMnBr}_{3}[\beta=0.25(1)]$ [45], $\mathrm{CsCuCl}_{3}[\beta=0.25(2)]$ [46], and $\mathrm{CsNiCl}_{3}[\beta=0.243(5)]$ [47] even though all of them exhibit the magnetic long-range order already at ambient pressure, suggesting the absence of pressure-induced QPT, and inaccessibility to the QCP.

The $X Y$ STA model is characterized by the continuous $U(1)$ and discrete $Z_{2}$ symmetries, i.e., $U(1) \times Z_{2}$ symmetry $[42,43]$. In this symmetry, it is predicted that a chiral liquid state $[31,48,49]$, in which only the $Z_{2}$ symmetry is broken and the vector chirality $\kappa_{i j}=S_{i} \times S_{j}$ is ordered, emerges on the side of the quantum disordered phase. The chiral liquid state can be detected by the measurement of dielectric property because the vector chirality is related to magnetoelectric effect based on the spin current mechanism [50-52]. The chiral liquid state in the $X Y$ STA model has not been observed in $\mathrm{CsMnBr}_{3}$, $\mathrm{CsCuCl}_{3}$, and $\mathrm{CsNiCl}_{3}$, which exhibit the magnetic long-range order at ambient pressure. $\mathrm{CsFeCl}_{3}$ is thus a candidate that is accessible to the chiral liquid state in the vicinity of the QCP in the disordered phase.

In conclusion the $120^{\circ}$ structure with the propagation vector of $\boldsymbol{k}_{\text {mag }}=(1 / 3,1 / 3,0)$ was evidenced in the pressure-induced ordered phase of $\mathrm{CsFeCl}_{3}$ by the combination of the neutron diffraction experiments under pressures and magnetic structure analysis. The critical exponent of the order parameter was estimated to be $\beta=0.24(1)$, which was consistent with that for the $X Y$ STA model $\beta=0.253$. This criticality belongs to 
the universality class $U(1) \times Z_{2}$ in which the chiral liquid state is predicted. The study on the spin dynamics under pressure has a chance to observe a nontrivial magnon in the vicinity of the QCP in the frustrated triangular lattice for a future perspective.

We thank to D. Cheptiakov, J. S. White, and S. Hasegawa for supporting the pressure experiment at PSI. We are also grateful to T. Okubo for helpful discussion. Travel expense for the neutron diffraction experiments performed using ZEBRA at
PSI, Switzerland was supported by the General User Program for Neutron Scattering Experiments, Institute for Solid State Physics, The University of Tokyo (Proposal No. 17515), at JRR-3, Japan Atomic Energy Agency, Tokai, Japan. The neutron scattering experiment at the HRC spectrometer in JPARC/MLF was approved by the Neutron Scattering Program Advisory Committee of IMSS, KEK (Proposals No. 2015S01, No. 2016S01, and No. 2017S01) and ISSP. S.H. was supported by the Japan Society for the Promotion of Science through the Leading Graduate Schools (MERIT).
[1] S. Sachdev, Quantum Phase Transitions, 2nd ed. (Cambridge University Press, Cambridge, UK, 2011).

[2] S. Sachdev, Science 288, 475 (2000).

[3] A. Koga and N. Kawakami, Phys. Rev. Lett. 84, 4461 (2000).

[4] T. Giamarchi, Ch. Rüegg, and O. Tchernyshyov, Nat. Phys. 4, 198 (2008).

[5] E. Dagotto, Rev. Mod. Phys. 66, 763 (1994).

[6] J. Orenstein and A. J. Millis, Science 288, 468 (2000).

[7] J. Paglione and R. L. Greene, Nat. Phys. 6, 645 (2010).

[8] T. Shibauchi, A. Carrington, and Y. Matsuda, Annu. Rev. Condens. Matter Phys. 5, 113 (2014).

[9] P. Gegenwart, Q. Si, and F. Steglich, Nat. Phys. 4, 186 (2008).

[10] Q. Si and F. Steglich, Science 329, 1161 (2010).

[11] B. Spivak, S. V. Kravchenko, S. A. Kivelson, and X. P. A. Gao, Rev. Mod. Phys. 82, 1743 (2010).

[12] M. Greiner, O. Mandel, T. Esslinger, T. W. Hänsch, and I. Bloch, Nature (London) 415, 39 (2002).

[13] I. Bloch, J. Dalibard, and W. Zwerger, Rev. Mod. Phys. 80, 885 (2008).

[14] A. Oosawa, M. Ishii, and H. Tanaka, J. Phys.: Condens. Matter 11, 265 (1999).

[15] H. Tanaka, A. Oosawa, T. Kato, H. Uekusa, Y. Ohashi, K. Kakurai, and A. Hoser, J. Phys. Soc. Jpn. 70, 939 (2001).

[16] A. Oosawa, M. Fujisawa, T. Osakabe, K. Kakurai, and H. Tanaka, J. Phys. Soc. Jpn. 72, 1026 (2003).

[17] K. Goto, M. Fujisawa, T. Ono, H. Tanaka, and Y. Uwatoko, J. Phys. Soc. Jpn. 73, 3254 (2004).

[18] Ch. Rüegg, A. Furrer, D. Sheptyakov, Th. Strässle, K. W. Krämer, H.-U. Güdel, and L. Mélési, Phys. Rev. Lett. 93, 257201 (2004).

[19] P. Merchant, B. Normand, K. W. Krämer, M. Boehm, D. F. McMorrow, and Ch. Rüegg, Nat. Phys. 10, 373 (2014).

[20] T. Nikuni, M. Oshikawa, A. Oosawa, and H. Tanaka, Phys. Rev. Lett. 84, 5868 (2000).

[21] M. Matsumoto, B. Normand, T. M. Rice, and M. Sigrist, Phys. Rev. B 69, 054423 (2004).

[22] A. Khudoyberdiev, A. Rakhimov, and A. Schilling, New J. Phys. 19, 113002 (2017).

[23] Y. Kulik and O. P. Sushkov, Phys. Rev. B 84, 134418 (2011).

[24] Y. Q. Qin, B. Normand, A. W. Sandvik, and Z. Y. Meng, Phys. Rev. Lett. 118, 147207 (2017).

[25] J. Oitmaa, Y. Kulik, and O. P. Sushkov, Phys. Rev. B 85, 144431 (2012).

[26] S. Jin and A. W. Sandvik, Phys. Rev. B 85, 020409(R) (2012).

[27] M.-T. Kao and F.-J. Jiang, Eur. Phys. J. B 86, 419 (2013).

[28] D.-R. Tan and F.-J. Jiang, Phys. Rev. B 95, 054435 (2017).
[29] H. D. Scammell and O. P. Sushkov, Phys. Rev. B 95, 094410 (2017).

[30] M. Matsumoto and M. Koga, J. Phys. Soc. Jpn. 76, 073709 (2007).

[31] Z. Wang, A. E. Feiguin, W. Zhu, O. A. Starykh, A. V. Chubukov, and C. D. Batista, Phys. Rev. B 96, 184409 (2017).

[32] A. Kohne, E. Kemnitz, H. Mattausch, and A. Simon, Z. Kristallogr. 203, 316 (1993).

[33] H. Y. W. Kozukue and K. Hirakawa, J. Phys. Soc. Jpn. 49, 144 (1980).

[34] P. A. Montano, H. Shechter, E. Cohen, and J. Makovsky, Phys. Rev. B 9, 1066 (1974).

[35] N. Kurita and H. Tanaka, Phys. Rev. B 94, 104409 (2016).

[36] J. Rodriguez-Carvajal, Physica B 192, 55 (1993).

[37] A. S. Wills, Physica B 276-278, 680 (2000).

[38] H. Nakao, M. Nishi, Y. Fujii, T. Masuda, I. Tsukada, K. Uchinokura, K. Hirota, and G. Shirane, J. Phys. Soc. Jpn. 68, 3662 (1999).

[39] Y. J. Wang, V. Kiryukhin, R. J. Birgeneau, T. Masuda, I. Tsukada, and K. Uchinokura, Phys. Rev. Lett. 83, 1676 (1999).

[40] D. M. Pajerowski, C. R. Rotundu, J. W. Lynn, and R. J. Birgeneau, Phys. Rev. B 87, 134507 (2013).

[41] M. G. Kim, M. Wang, G. S. Tucker, P. N. Valdivia, D. L. Abernathy, S. Chi, A. D. Christianson, A. A. Aczel, T. Hong, T. W. Heitmann, S. Ran, P. C. Canfield, E. D. Bourret-Courchesne, A. Kreyssig, D. H. Lee, A. I. Goldman, R. J. McQueeney, and R. J. Birgeneau, Phys. Rev. B 92, 214404 (2015).

[42] H. Kawamura, J. Phys. Soc. Jpn. 61, 1299 (1992).

[43] H. Kawamura, J. Phys.: Condens. Matter 10, 4707 (1998).

[44] M. Campostrini, M. Hasenbusch, A. Pelissetto, P. Rossi, and E. Vicari, Phys. Rev. B 63, 214503 (2001).

[45] Y. Ajiro, T. Nakashima, Y. Unno, H. Kadowaki, M. Mekata, and N. Achiwa, J. Phys. Soc. Jpn. 57, 2648 (1988).

[46] U. Schotte, N. Stusser, K. D. Schotte, H. Weinfurter, H. M. Mayer, and M. Winkelmann, J. Phys.: Condens. Matter 6, 10105 (1994).

[47] M. Enderle, R. Schneider, Y. Matsuoka, and K. Kakurai, Physica B 234, 554 (1997).

[48] A. V. Chubukov and O. A. Starykh, Phys. Rev. Lett. 110, 217210 (2013).

[49] E. Parker and L. Balents, Phys. Rev. B 95, 104411 (2017).

[50] H. Katsura, N. Nagaosa, and A. V. Balatsky, Phys. Rev. Lett. 95, 057205 (2005).

[51] I. A. Sergienko and E. Dagotto, Phys. Rev. B 73, 094434 (2006).

[52] M. Mostovoy, Phys. Rev. Lett. 96, 067601 (2006). 